\title{
Integer and half-integer quantum Hall effect in silicene: Influence of an external electric field and impurities
}

\author{
Kh. Shakouri, ${ }^{1}$ P. Vasilopoulos, ${ }^{2}$ V. Vargiamidis, ${ }^{2}$ and F. M. Peeters ${ }^{1}$ \\ ${ }^{1}$ Departement Fysica, Universiteit Antwerpen Groenenborgerlaan 171, B-2020 Antwerpen, Belgium \\ ${ }^{2}$ Department of Physics, Concordia University, 7141 Sherbrooke Ouest Montréal, Québec H4B 1R6, Canada
}

(Received 2 October 2014; published 15 December 2014)

\begin{abstract}
The influence of silicene's strong spin-orbit interaction and of an external electric field $E_{z}$ on the transport coefficients are investigated in the presence of a perpendicular magnetic field $B$. For finite $E_{z}$ the spin and valley degeneracy of the Landau levels is lifted and leads to additional plateaus in the Hall conductivity, at half-integer values of $4 e^{2} / h$, due to spin intra-Landau-level transitions that are absent in graphene. These plateaus are more sensitive to disorder and thermal broadening than the main plateaus, occurring at integral values of $4 e^{2} / h$, when the Fermi level passes through the Landau levels. We also evaluate the Hall and longitudinal resistivities and critically contrast the results with those for graphene on a substrate.
\end{abstract}

DOI: 10.1103/PhysRevB.90.235423

PACS number(s): 73.20.-r, 73.43.-f, 72.10.-d

\section{INTRODUCTION}

Recent progress in the exfoliation of bulk layered materials has facilitated the realization of ultrathin two-dimensional (2D), one-atom-thick layers that exhibit novel properties. The silicon analog of graphene, called silicene, is a new 2D material that has been predicted to be stable [1] and has currently been synthesized on a $\mathrm{Ag}(111)$ or $\mathrm{MoS}_{2}$ surface [2,3]. Though there is some controversy as to how its free-standing version can be really synthesized [4], it has attracted a great deal of attention [5-7] because, contrary to graphene, it has a strong spin-orbit interaction (SOI). In addition, the Dirac cones in silicene are similar to those of graphene. This similarity results from the fact that carbon and silicon belong to the same column in the periodic table of elements.

The strong SOI of silicene is predicted to open a gap with a width of approximately $1.55 \mathrm{meV}$ [5] between the low-energy Dirac-like cones, which is appropriate for the observation of the quantum spin-Hall effect. It has also been predicted that the created gap can be tuned [6] by applying an external electric field $E_{z}$ perpendicular to the silicene sheet. The tunability of the band gap is a consequence of the buckled structure, with one of the two sublattices of the honeycomb lattice shifted vertically with respect to the other [see Fig. 1(a)]. Recent density-functional theory calculations predicted that silicene should exhibit a quantum spin-Hall effect at an accessible temperature [5]. These and many other properties, reviewed in Ref. [7], together with silicene's compatibility with siliconbased nanoelectronic technology, make silicene a promising material for applications. Encouraging in this direction is the very recently reported local formation of high-buckled silicene nanosheets on a $\mathrm{MoS}_{2}$ surface [3].

So far most of the works on silicene study its properties in the absence of a magnetic field $B$. But it is of interest to know how silicene's strong SOI affects its magnetotransport properties. One might expect that the results are similar to those for graphene on a substrate [8,9], which creates a gap $\Delta$ in the single-particle spectrum, but this is not the case. The reason is that the equivalent quantity to $\Delta$ in silicene depends on the spin and valley degrees of freedom [10] when a perpendicular electric field $E_{z}$ is present.
Our previous work [10] pertains only to periodically modulated silicene in very weak magnetic fields and addresses the commensurability oscillations, but almost neglects the Shubnikov-de Haas oscillations that usually start at $B \approx 0.6 \mathrm{~T}$. We now address the effect of a homogeneous perpendicular electric field on silicene's magnetotransport properties when a strong quantizing magnetic field is present. We derive an analytic expression for the Hall conductivity and show that the application of a perpendicular electric field $E_{z}$ doubles the Shubnikov-de Haas oscillations, resulting in integer and half-integer conductivity plateaus. We also evaluate the Hall and longitudinal resistivities and critically contrast the results with those for graphene on a substrate.

In Sec. II we present one-electron properties and the relevant conductivities. The evaluation of the latter is carried out in some detail in Sec. III. In Sec. IV we summarize our results. Finally, in the Appendix we outline the derivation of the Hall conductivity.

\section{FORMALISM}

\section{A. One-electron attributes}

The effective Hamiltonian of buckled structures of groupIV A elements such as silicene has already been derived $[6,11]$ by combining first-principles calculations with a tight-binding treatment. Since the nearest- and next-nearestneighbor Rashba SOIs, denoted as $\lambda_{R 1}$ and $\lambda_{R 2}$ in Ref. [6], are negligible compared to the intrinsic SOI, $\lambda_{\text {so }}$, we omit them in the calculations. Using this assumption one renders the $4 \times 4$ matrix Hamiltonian into two $2 \times 2$ block-diagonal ones, in terms of the spin index $s_{z}= \pm 1$ (for an analytic justification see, e.g., Ref. [12]), whose form in the presence of a magnetic field $B$ is

$$
H_{\xi}=v_{F}\left[\tau_{x} \Pi_{x}-\xi \tau_{y} \Pi_{y}\right]-\left(\xi s_{z} \lambda_{\mathrm{so}}-e \ell E_{z}\right) \tau_{z} .
$$

Here $v_{F}$ is the Fermi velocity, $\tau_{i}(i=x, y, z)$ the corresponding Pauli matrices of the sublattice pseudospin, $2 \ell$ the vertical distance between the two sublattices, $\xi= \pm 1$ the valley index, and $\Pi=\mathbf{p}+e \mathbf{A}$. Substituting the $\tau_{i}$ matrices in Eq. (1) 

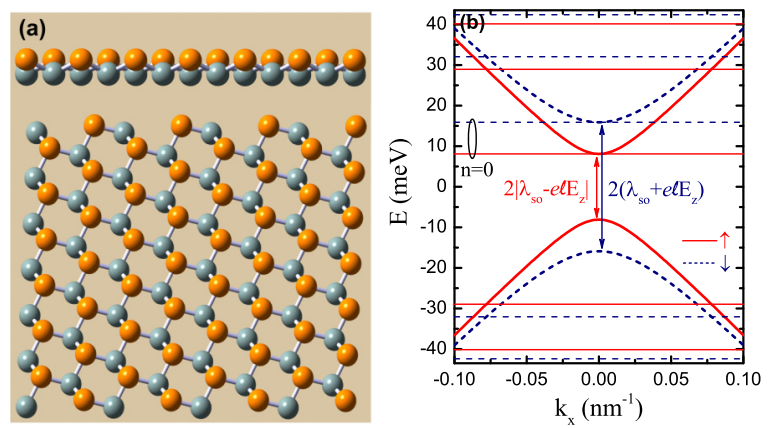

FIG. 1. (Color online) (a) Buckled structure of silicene. (b) The spin-split energy spectrum of silicene, for the $K$ valley, subject to a perpendicular electric field $E_{z}=12 \mathrm{mV} / \ell$. The bold solid (dashed) curves indicate the energy dispersion in the absence of a magnetic field for spins up (down). The horizontal lines show the split LLs for $B=2 \mathrm{~T}$.

yields

$$
H_{\xi}=\left[\begin{array}{cc}
e_{s_{z}, \xi} & v_{F} \Pi_{ \pm} \\
v_{F} \Pi_{\mp} & -e_{s_{z}, \xi}
\end{array}\right],
$$

where $e_{s_{z}, \xi}=-\xi \lambda_{\mathrm{so}} s_{z}+e \ell E_{z}$ and $\Pi_{ \pm}=\Pi_{x} \pm i \Pi_{y}$. Equation (2) is identical in form with Eq. (1) of Ref. [8] and Eq. (2) of Ref. [9] that describe graphene on a substrate: simply $e_{0}$ of Ref. [8] or $\Delta$ of Ref. [9] is replaced by the tunable parameter $e_{s_{z}, \xi}$ that depends on $\xi, s_{z}$, and $E_{z}$ whereas $e_{0}$ and $\Delta$ do not. The most straightforward consequence of this dependence is that the band gap between the conduction and valence bands is tunable by the electric field $E_{z}$, as shown in Fig. 1(b), and more importantly the gap size is different for opposite spins [13].

Taking the magnetic field perpendicular to the silicene sheet, $\vec{B}=B \hat{e}_{z}$, we can write the vector potential in the Landau gauge $\vec{A}=(-B y, 0,0)$, use the wave function in the ansatz $\Psi(x, y)=e^{i k_{x} x} \psi(y) / \sqrt{L_{x}}$, and obtain the kinetic momentum as $\pi_{ \pm}=\hbar\left(k_{x} \pm \partial_{y}-y / \ell_{c}^{2}\right)$ where $\ell_{c}=\sqrt{\hbar / e B}$ is the magnetic length. Then, the eigenvectors and eigenvalues of Eq. (2) are obtained in a standard manner by solving the secular equation $(H-E I) \Psi=0$, where $I$ is the identity matrix. The eigenvalues are

$$
E_{n s_{z}}^{\lambda \xi}=\lambda\left[\hbar^{2} \omega_{c}^{2} n+e_{s_{z}, \xi}^{2}\right]^{1 / 2},
$$

where $\omega_{c}=\sqrt{2} v_{F} / \ell_{c}$ is the cyclotron frequency; the nonnegative integer $n$ labels the Landau levels (LLs) and the factor $\lambda=1(-1)$ the electron (hole) states for $n \geqslant 1$. Notice that for $n=0$ either $K$ or $K^{\prime}$ valley involves only one solution and the $\lambda$ dependence disappears:

$$
E_{0 s_{z}}^{\xi}=\xi e_{s_{z}, \pm}
$$

For $\xi=1$ and $n \geqslant 1$ the eigenvectors $\Psi_{n s_{z}}^{\lambda \xi}\left(\mathbf{r}, k_{x}\right)$ are

$$
\Psi_{n s_{z}}^{\lambda 1}\left(\mathbf{r}, k_{x}\right)=\frac{1}{\sqrt{2 L_{x}}}\left(\begin{array}{c}
\eta_{+} \Phi_{n}(v) \\
-\lambda \eta_{-} \Phi_{n-1}(v)
\end{array}\right) e^{i k_{x} x} .
$$

$\Phi_{n}(v)$ are the usual normalized oscillator functions, $v=$ $y / \ell_{c}-\ell_{c} k_{x}, \quad \eta_{ \pm}=[1 \pm \cos \theta]^{1 / 2}$, and $\cos \theta=e_{s_{z},+} / E_{n s_{z}}^{\lambda \xi}$. The eigenvector for the $n=0 \mathrm{LL}$ is given by $\Psi_{0 s_{z}}^{\xi=1}=$ $\left[\Phi_{0}(v), 0\right]^{T} e^{i k_{x} x} / \sqrt{L_{x}}$ with $T$ denoting the transpose. The
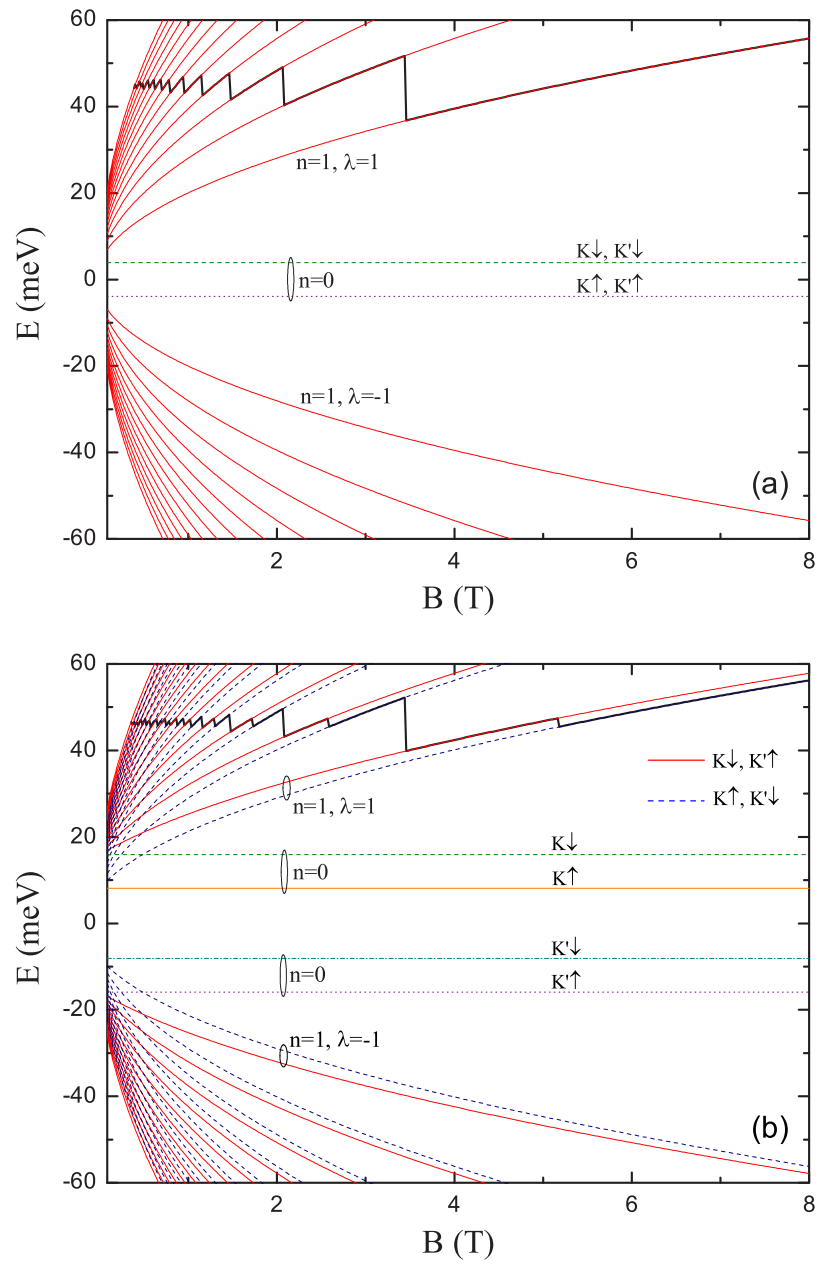

FIG. 2. (Color online) Energy spectrum $E_{n s_{z}}^{\lambda \xi}$ as a function of the magnetic field $B$. Panel (a) is for $E_{z}=0$ and panel (b) for $E_{z}=$ $12 \mathrm{mV} / \ell$. The black solid curve shows the Fermi level vs $B$. The additional intra- $L L$ small jumps in (b) result from the lifting of the spin degeneracy in each valley; e.g., for the $K$ valley, the solid and dashed curves are for spin down and spin up, respectively, whereas for the $K^{\prime}$ valley they are interchanged. Notice also the lifting of the spin and valley degeneracy for the $n=0 \mathrm{LL}$.

eigenvectors for $\xi=-1$ are given by Eq. (5) but with $\Phi_{n}(v)$ and $\Phi_{n-1}(v)$ interchanged and $e_{S_{z}},+$ replaced by $-e_{s_{z},-}$. In a similar manner one obtains $\Psi_{0 s_{z}}^{\xi=-1}$.

The LLs given by Eqs. (3) and (4) are spin and valley dependent via $e_{s_{z}, \pm}$, but independent of $k_{x}$ as seen in Fig. 2(b), so that by applying a perpendicular homogeneous electric field the spin degeneracy of all LLs is lifted. Meanwhile, the $n=0 \mathrm{LL}$ is always twice less degenerate than the $n \geqslant 1 \mathrm{LLs}$ irrespective of whether the field $E_{z}$ is present or not. To better appreciate the difference between the $E_{z}=0$ and $E_{z} \neq 0$ cases we plot the spectrum in Fig. 2 versus the magnetic field $B$. Panels (a) and (b) correspond to $E_{z}=0$ and $E_{z}=12 \mathrm{mV} / \ell$, respectively. The black solid curve shows the Fermi level vs $B$. The additional intra- $L L$ small jumps in (b) result from the lifting of the spin degeneracy; the solid and dashed curves $(n \geqslant 1)$ are, respectively, for spin down and spin up in the $K$ valley, respectively. For the $K^{\prime}$ valley the spin is reversed; e.g., for $n \geqslant 1$, the spin-up electrons in the $K$ valley have the same 


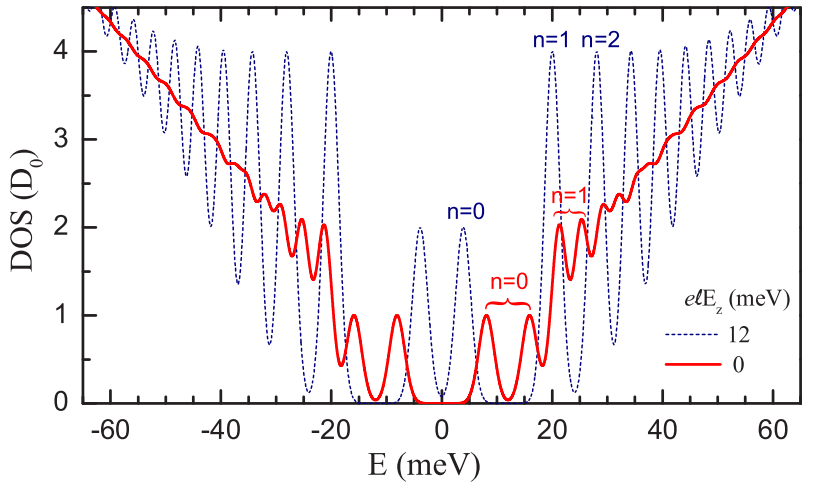

FIG. 3. (Color online) Density of states $D(E)$ as a function of the energy for $B=1 \mathrm{~T}$ and $\Gamma=0.1 \hbar \omega_{c}$. The index $n$ above the peaks labels the LLs.

energy as the spin-down ones in the $K^{\prime}$ valley. For $n \geqslant 1$ all LLs in (a) are fourfold degenerate corresponding to the two valley and two spin states except for the split $n=0 \mathrm{LL}$ which is twice degenerate. But in (b) the presence of $E_{z}$ splits the LLs and reduces twice the degeneracy. Another feature worth noticing is the lifting of the spin and valley degeneracies for the $n=0$ LL.

The spectrum given by Eq. (3) leads to the density of states $D(E)$, per unit area $S_{0}$,

$$
D(E)=\frac{1}{\pi S_{0}} \operatorname{Im} \sum_{\zeta} \frac{1}{E-E_{\zeta}+i \Gamma},
$$

where $\zeta$ is a concise symbol for the $n, s_{z}, \lambda, \xi$, and $k_{x}$ quantum numbers and $\Gamma$ the width of the broadened LLs due to scattering, which for convenience is taken independent of $\zeta$. For scattering by $\delta$-function impurities the broadening $\Gamma$ is equal [14] to $\sqrt{2 / A}$, where $A$ is a dimensionless parameter inversely proportional to the impurity density $n_{i}$ and strength $V_{0}$ of the scattering potential, $A=4 \pi \hbar^{2} v_{F}^{2} / n_{i} V_{0}^{2}$. We plot $D(E)$ vs $E$ in Fig. 3 for $A=150$, corresponding to $\Gamma \approx$ $0.1 \hbar \omega_{c}$, and $B=1 \mathrm{~T}$. The solid curve is for $E_{z}=0$ and the dotted one for $e \ell E_{z}=12 \mathrm{meV}$. As can be seen, the overall shape of the peaks is similar to that for graphene, but a finite $E_{z}$ lifts the spin degeneracy of the LLs, which is absent in graphene, and doubles their number. These peaks become very sharp if we neglect the LL broadening.

Equations (3)-(5) are necessary for the evaluation of the conductivities. For that we also need the off-diagonal matrix elements of the components $v_{x}$ and $v_{y}$ of the velocity operator $\boldsymbol{v}$ obtained from Eq. (1) and the general formula $\boldsymbol{v}=\partial H / \hbar \partial \mathbf{k}$. We find $v_{x}=v_{F} \sigma_{x}$ and $v_{y}=-\xi v_{F} \sigma_{y}$. Then using the basis (3) gives

$$
\begin{aligned}
& \left\langle\lambda, n, s_{z}, k_{x}\left|v_{x}\right| \lambda^{\prime}, n^{\prime}, s_{z}^{\prime}, k_{x}^{\prime}\right\rangle \\
& \quad=\left(v_{F} / 2\right) \delta_{k_{x}, k_{x}^{\prime}} \delta_{s_{z} s_{z}^{\prime}}\left[\lambda \eta_{+} \eta_{-}^{\prime} \delta_{n, n^{\prime}-1}+\lambda^{\prime} \eta_{-} \eta_{+}^{\prime} \delta_{n-1, n^{\prime}}\right], \\
& \left\langle\lambda^{\prime}, n^{\prime}, s_{z}^{\prime}, k_{x}^{\prime}\left|v_{y}\right| \lambda, n, s_{z}, k_{x}\right\rangle \\
& \quad=\left(i v_{F} / 2\right) \delta_{k_{x}, k_{x}^{\prime}} \delta_{s_{z} s_{z}^{\prime}}\left[\lambda^{\prime} \eta_{+}^{\prime} \eta_{-} \delta_{n^{\prime}, n-1}-\lambda \eta_{-}^{\prime} \eta_{+} \delta_{n, n^{\prime}-1}\right] .
\end{aligned}
$$

The factor $\delta_{s_{z} s_{z}^{\prime}}$ is due to the fact that in each block, in which $H_{\xi}$ is diagonalized and the basis (5) is found, the spin $s_{z}$ does not change.

\section{B. Linear-response conductivity expressions}

In linear response theory, the responses to external timedependent perturbations can be expressed in terms of the unperturbed system functions. More generally, the manybody Hamiltonian of the system can be described by $H=$ $H_{0}+H_{I}-\mathbf{R} \cdot \mathbf{F}(t)$, where $H_{0}$ is the unperturbed part, $H_{I}$ involves binary interactions between electrons with impurities or phonons, $-\mathbf{R} \cdot \mathbf{F}(t)$ is the interaction of the system with an external time-dependent field $\mathbf{F}(t), \mathbf{R}=\sum_{\mathbf{r}_{i}}$, and $\mathbf{r}_{i}$ is the position operator $\mathbf{r}_{i}$ of electron $i$. In order to evaluate transport coefficients such as electrical conductivity we adopt the formalism developed in Ref. [15] by using $\mathbf{F}(t)=e \mathbf{E}(t)$, where $\mathbf{E}(t)$ is the electric field. For a certain representation in which $H_{0}$ is diagonal the many-body density operator $\rho=\rho^{d}+\rho^{n d}$ has diagonal $\left(\rho^{d}\right)$ and nondiagonal $\left(\rho^{n d}\right)$ parts. Accordingly, for weak electric fields and weak scattering potentials when first-order Born approximation is admissible, the corresponding conductivity tensor has diagonal $\left(\sigma_{\mu \nu}^{d}\right)$ and nondiagonal $\left(\sigma_{\mu \nu}^{n d}\right)$ parts: $\sigma_{\mu \nu}=\sigma_{\mu \nu}^{d}+\sigma_{\mu \nu}^{n d}, \mu, v=x, y$. In general two mechanisms contribute to the current, diffusion, and hopping, but usually only one of them is present. When a magnetic field is present, we have only the hopping contribution since the diffusive one, involving only the diagonal elements of the velocity operator, vanishes identically on account of Eqs. (7) and (8). This hopping contribution is given by

$$
\sigma_{x x}^{\mathrm{col}}=\frac{\beta e^{2}}{2 S_{0}} \sum_{\zeta, \zeta^{\prime}} f\left(E_{\zeta}\right)\left[1-f\left(E_{\zeta^{\prime}}\right)\right] W_{\zeta \zeta^{\prime}}\left(y_{\zeta}-y_{\zeta^{\prime}}\right)^{2},
$$

where $f_{\zeta}=\left[1+\exp \beta\left(E_{\zeta}-E_{F}\right)\right]^{-1}$ is the Fermi-Dirac distribution function, $\beta=1 / k_{B} T, T$ the temperature, and $E_{F}$ the Fermi level. Further, $W_{\zeta \zeta^{\prime}}$ is the transition rate between the states $|\zeta\rangle$ and $\left|\zeta^{\prime}\right\rangle$ and $y_{\zeta}=\langle\zeta|y| \zeta\rangle$ the expectation value of the coordinate $y$. For elastic scattering by impurities the transition rate $W_{\zeta \zeta^{\prime}}$ is

$$
W_{\zeta \zeta^{\prime}}=\frac{2 \pi n_{i}}{\hbar S_{0}} \sum_{\mathbf{q}}|U(\mathbf{q})|^{2}\left|F_{\zeta \zeta^{\prime}}(u)\right|^{2} \delta\left(E_{\zeta}-E_{\zeta^{\prime}}\right) \delta_{k_{x}, k_{x}^{\prime}+q_{x}},
$$

with $n_{i}$ the concentration of impurities and $\mathbf{q}$ the change in electron wave vector. Further, $u=\ell_{c}^{2} q^{2} / 2, U(\mathbf{q})$ is the Fourier transform of the scattering potential, and $F_{\zeta \zeta^{\prime}}(u)$ the form factor for $\left\langle\zeta\left|e^{i \mathbf{q} \cdot \mathbf{r}}\right| \zeta^{\prime}\right\rangle$. The expectation value $y_{\zeta}$ is readily evaluated: one finds $y_{\zeta}=\ell_{c}^{2} k_{x}$ and $y_{\zeta^{\prime}}=\ell_{c}^{2} k_{x}^{\prime}$.

Regarding the contribution $\sigma_{\mu \nu}^{\text {nd }}$ one can use the identity $f_{\zeta}\left(1-f_{\zeta^{\prime}}\right)\left[1-\exp \beta\left(E_{\zeta}-E_{\zeta^{\prime}}\right)\right]=f_{\zeta}-f_{\zeta^{\prime}}$ and cast $[16,17]$ the original expression of Ref. [15] in the familiar form

$$
\sigma_{\mu \nu}^{n d}(0)=\frac{i \hbar e^{2}}{S} \sum_{\zeta \neq \zeta^{\prime}} \frac{\left(f_{\zeta}-f_{\zeta^{\prime}}\right) v_{v \zeta \zeta^{\prime}} v_{\mu \zeta^{\prime} \zeta}}{\left(E_{\zeta}-E_{\zeta^{\prime}}\right)\left(E_{\zeta}-E_{\zeta^{\prime}}+i \Gamma_{\zeta}\right)},
$$

where $v_{\nu \zeta \zeta^{\prime}}$ and $v_{\mu \zeta \zeta^{\prime}}$ are the nondiagonal matrix elements of the velocity operator, $\mu, v=x, y$. The sum runs over all quantum numbers of the states $|\zeta\rangle=\left|n, s_{z}, \lambda, \xi, k_{x}\right\rangle$ and $\left|\zeta^{\prime}\right\rangle=$ $\left|n^{\prime}, s_{z}^{\prime}, \lambda^{\prime}, \xi^{\prime}, k_{x}^{\prime}\right\rangle$ provided $|\zeta\rangle \neq\left|\zeta^{\prime}\right\rangle$. The infinitesimal quantity $\epsilon$ in the original form [15] has been replaced by $\Gamma_{\zeta}$ to account for the broadening of the energy levels. 


\section{RESULTS}

\section{A. Hall conductivity}

Assuming that the broadening of the LLs is approximately the same for all states, $\Gamma_{\zeta} \approx \Gamma$, one can show that the imaginary part of Eq. (11) vanishes. In the spirit of the original derivation of Eq. (11) and to obtain a transparent result for $\sigma_{y x}$ we first take $\Gamma=0$. We will consider a finite $\Gamma$ later in the evaluation of the longitudinal conductivity.

To proceed, one needs the product of the velocity matrix elements in each valley for $v=x$ and $\mu=y$ :

$$
P_{n n^{\prime}, s_{z} s_{z}^{\prime}}^{\lambda \lambda^{\prime} k_{x}^{\prime}}=\left\langle\lambda n s_{z} k_{x}\left|v_{x}\right| \lambda^{\prime} n^{\prime} s_{z}^{\prime} k_{x}^{\prime}\right\rangle\left\langle\lambda^{\prime} n^{\prime} s_{z}^{\prime} k_{x}^{\prime}\left|v_{y}\right| \lambda n s_{z} k_{x}\right\rangle .
$$

Since the matrix elements (7) and (8) are diagonal in $k_{x}$, henceforth we suppress the quantum number $k_{x}$ in order to simplify the notation. After an explicit evaluation of the product $P_{n n^{\prime}, s_{z} s_{z}^{\prime}}^{\lambda \lambda \prime^{\prime}}$, for the $K$ valley one obtains

$$
P_{n n^{\prime}, s_{z} s_{z}^{\prime}}^{\lambda \lambda^{\prime}}=\frac{i v_{F}^{2}}{4}\left[\eta_{+}^{\prime 2} \eta_{-}^{2} \delta_{n-1, n^{\prime}}-\eta_{+}^{2} \eta_{-}^{\prime 2} \delta_{n, n^{\prime}-1}\right] \delta_{s_{z} s_{z}^{\prime}}
$$

As usual the matrix elements between the zeroth LL and the other LLs should be treated separately since the superscripts $\lambda$ and $\lambda^{\prime}$ are not present for $n=0$ [17]. Corresponding to Eqs. (12) and (13) one gets

$$
P_{0 n^{\prime}}^{s_{z} s_{z}^{\prime}}=\frac{-i v_{F}^{2}}{2} \eta_{-}^{\prime 2} \delta_{n^{\prime}, 1} \delta_{s_{z} s_{z}^{\prime}}, \quad P_{n 0}^{s_{z} s_{z}^{\prime}}=\frac{i v_{F}^{2}}{2} \eta_{-}^{2} \delta_{n, 1} \delta_{s_{z} s_{z}^{\prime}} .
$$

Notice that we omitted the complex modulus signs in the coefficients $\eta_{ \pm}^{2}$ since they are always real. Now, one needs to sum over all possible combinations of the matrix elements, and for simplicity we first start with $\sum_{\lambda \lambda^{\prime}}=$ $\sum_{++}+\sum_{--}+\sum_{+-}+\sum_{-+}$. Here the subscript $+/-$denotes the conduction/valence band and only the $n>0$ LLs are considered due to the peculiarity of the zeroth level. As shown in the Appendix, the $K$ valley's contribution to the Hall conductivity, $\sigma_{y x}^{K}$, can be expressed as a sum of two terms: one in which the prefactor is independent of $e_{s_{z},+}$ and a term in which it is linear in $e_{s_{z},+}$, i.e., $\sigma_{y x}^{K}=\sigma_{K}+\delta \sigma_{K}$, with

$$
\sigma_{K}=\frac{e^{2}}{h} \sum_{n=1, s_{z}}\left(n+\frac{1}{2}\right)\left(f_{n s_{z}}^{+}+f_{n s_{z}}^{-}-f_{n+1 s_{z}}^{+}-f_{n+1 s_{z}}^{-}\right)
$$

and

$$
\delta \sigma_{K}=\frac{e^{2}}{2 h} \sum_{n=1, s_{z}} e_{s_{z},+}\left[\frac{f_{n s_{z}}^{+}-f_{n s_{z}}^{-}}{\epsilon_{n s_{z}}}-\frac{f_{n+1 s_{z}}^{+}-f_{n+1 s_{z}}^{-}}{\epsilon_{n+1 s_{z}}}\right] .
$$

The sums in Eqs. (15) and (16) run from $n=1$ to infinity and the zeroth level should be added separately. Similarly, one can derive the same expressions for carriers in the vicinity of the $K^{\prime}$ point by replacing only $e_{s_{z}},+$ with $-e_{s_{z},-}$ everywhere. Then writing explicitly the sum over $s_{z}$ one sees that the correction terms $\delta \sigma_{K}$ and $\delta \sigma_{K^{\prime}}$ cancel each other provided both valleys in the $n$th LL are occupied; evidently they do not cancel when only a certain spin or valley state is occupied.

For $n=0$ the contribution of the $K$ valley is

$$
\sigma_{0 K}=\frac{e^{2}}{h} \sum_{s_{z}}\left[f_{0 s_{z}}-\frac{1}{2}\left(f_{1 s_{z}}^{+}+f_{1 s_{z}}^{-}\right)+\frac{e_{s_{z},+}}{2 \epsilon_{1 s_{z}}}\left(f_{1 s_{z}}^{+}-f_{1 s_{z}}^{-}\right)\right] \text {, }
$$

and, as previously for the $K^{\prime}$ valley, one simply replaces $e_{s_{z}}$, by $-e_{s_{z},-}$ in Eq. (17) to obtain $\sigma_{0 K^{\prime}}$. Note again that the terms $e_{s_{z}, \pm}$ cancel each other when summing over valley contributions, $\sigma_{0 K}+\sigma_{0 K^{\prime}}$, because $e_{\uparrow(\downarrow),+}=e_{\downarrow(\uparrow),-}$. This reveals that the conductivity $\sigma_{y x}$ is eventually independent of $e_{s_{z}, \pm}$; that is, the size of the band gap does not affect the transverse conductivity. Although this limit agrees with the results of Ref. [9] for graphene on a hexagonal boron nitride ( $\mathrm{hBN}$ ) substrate, a question still remains open: What is the difference between silicene and gapped graphene considering that the terms $e_{s_{z}, \pm}$ vanish explicitly in the expression of $\sigma_{y x}$ ? The answer lies in the Fermi function $f_{n s_{z}}$. To elucidate this, suppose that the hole states are already occupied and $f_{n s_{z}}^{-}=1$. Then the term $f_{n s_{z}}^{-}-f_{n+1 s_{z}}^{-}$in Eq. (15) vanishes, and we can use only the valley index $\xi$ as a superscript. The contribution of the two valleys is then accounted for in the following expression:

$$
\sigma_{y x}=\sum_{\xi} \sigma_{0 \xi}+\frac{e^{2}}{h} \sum_{n=1}^{\infty}\left(n+\frac{1}{2}\right) \sum_{s_{z}, \xi}\left(f_{n s_{z}}^{\xi}-f_{n+1 s_{z}}^{\xi} .\right.
$$

At zero or very low temperatures the last sum in Eq. (18) has the value 4 or 2 for $n=n_{F}$ depending on whether the number of filled spin-valley states is 4 or 2 , respectively. The latter occurs when the electric field splits the LLs and allows intra-LL jumps of $E_{F}$ between spin-valley states in the same Landau index $n_{F}$, cf. Fig. 2(b). But for graphene on an hBN substrate, the gap $\Delta$ corresponding to $e_{s_{z}, \pm}$ is constant and the fourfold degeneracy of the LLs makes this sum always equal to 4 because the occupation probability is equal for all spin-valley states. As a result, the additional small jumps of the Fermi level, clearly seen in the upper (black) curve in Fig. 4, lead to new steps in the Hall conductivity $\sigma_{y x}$ of silicene. In the main Fig. 4 we plot $\sigma_{y x}$ vs the magnetic field $B$ for $E_{z}=0$ (dashed curve) and $e \ell E_{z}=$ $12 \mathrm{meV}$ (solid curve), $T=\Gamma=0$, and with an electron density of $N_{e}=5 \times 10^{11} / \mathrm{cm}^{2}$. Notice also that Eq. (18) for graphene takes the form [18] $\sigma_{y x}^{n d}= \pm 4 e^{2} / h(n+1 / 2)$ which, in terms of the filling factor $v$, is written as $\sigma_{y x}^{n d}=v e^{2} / h$ with [19] $v=4(n+1 / 2)$. The additional factor $1 / 2$ is the most obvious manifestation of the anomalous integer quantum Hall effect

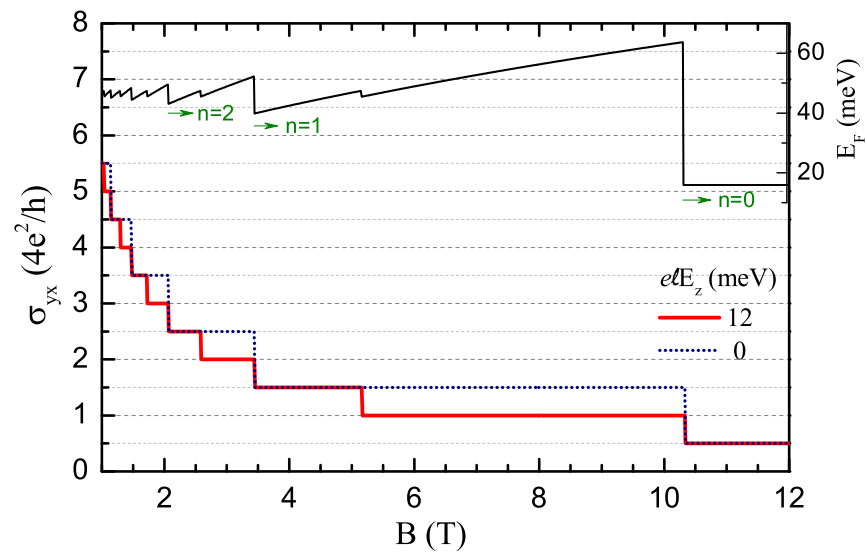

FIG. 4. (Color online) Hall conductivity vs magnetic field $B$ for $e \ell E_{z}=0$ (dashed curve) and $e \ell E_{z}=12 \mathrm{meV}$ (solid curve); the electron density is $n_{e}=5 \times 10^{11} / \mathrm{cm}^{2}$ and $T=\Gamma=0$. The upper curve shows the Fermi level vs $B$ and the small intra- $L L$ jumps in it signal transitions between the up and down spin states. 


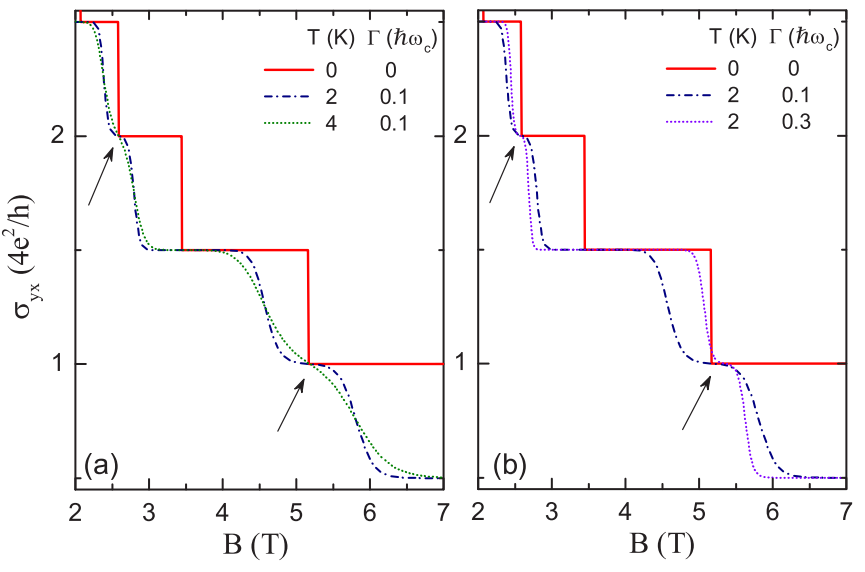

FIG. 5. (Color online) The same as in Fig. 4 but for $2 \leqslant B \leqslant 7 \mathrm{~T}$ and the red solid curve being the $T=\Gamma=0$ result of Fig. 4. Panel (a) is for different temperatures and panel (b) for different $\Gamma$ values.

(IQHE) in gapless, monolayer graphene. The existence of a Landau-like level at zero energy, for $\lambda_{\mathrm{so}}=E_{z}=0$, equally shared by the conduction and valence bands, is the key to understand the difference between the QHE in graphene and in common semiconductors such as GaAs [20].

Another notable feature of the new plateaus in $\sigma_{y x}$ is their dependence on the broadening $\Gamma$ and temperature. This is indicated by arrows in Fig. 5: the width of the additional plateaus caused by intra $L L$ transitions of the Fermi level becomes narrow with increasing temperature $T$ [Fig. 5(a)] or broadening $\Gamma$ [Fig. 5(b)]. By further increasing $T$ or $\Gamma$ the new plateaus disappear because the LL or thermal broadening of the Fermi function covers the spacing between the spin-split LLs. To "retain" these plateaus one has to use lower electron densities and apply a magnetic field for which the spin splitting exceeds the LL broadening $(\Gamma \propto \sqrt{B})$.

\section{B. Longitudinal conductivity and resistivity}

In a 2D electron system, a perpendicular magnetic field confines the electrons in quantized cyclotron orbits. At very low temperatures, when there is no external stimulation leading to electron diffusion, electron-impurity scattering can make electrons change their initial orbits and contribute to the longitudinal conductivity. We evaluate this contribution by modeling the impurity potential as that of a screened charge $U(\mathbf{r})=\left(e^{2} / 4 \pi \varepsilon_{r} \varepsilon_{0} r\right) e^{-k_{s} r}$, where $k_{s}$ is the screening wave vector, $\varepsilon_{r}$ the relative permittivity, and $\varepsilon_{0}$ the permittivity of vacuum. The Fourier transform of $U(\mathbf{r})$ is then given by $U(\mathbf{q})=U_{0} /\left(q^{2}+k_{s}^{2}\right)^{1 / 2}$, with $U_{0}=e^{2} /\left(2 \varepsilon_{r} \varepsilon_{0}\right)$. Further, if the impurity potential is strongly short-ranged (i.e., $\delta$-function type), one may use the approximation $k_{s} \gg q$ and obtain $U(\mathbf{q}) \approx U_{0} / k_{s}$. Upon inserting Eq. (10) in Eq. (9) one notices that the factor $\left(y_{\zeta}-y_{\zeta^{\prime}}\right)^{2}$ does not vanish, even for elastic collisions $E_{\zeta}=E_{\zeta^{\prime}}$. This is possible because the states $|\zeta\rangle$ and $\left|\zeta^{\prime}\right\rangle$ correspond to unequal wave vectors $k_{x}$ and $k_{x}^{\prime}=k_{x}-q_{x}$, respectively ( $q_{x}$ is the $x$ component of the change in wave vector q) and as a result the centers of the initial and final cyclotron orbits are different. Since the scattering is elastic and the eigenvalues are degenerate in the quantum number $k_{x}$, cf. Eq. (3), only the $n \rightarrow n$ transitions are allowed. In addition, no transition is allowed between the conduction and valence bands since the large band gap created by the strong SOI excludes elastic scattering. Then, using Eq. (3), one can replace the delta function in Eq. (10) by a Lorentzian of width $\Gamma$. Also, since in the transition $\zeta \rightarrow \zeta^{\prime}$ the quantum numbers $n, s_{z}, \lambda$, and $\xi$ remain unchanged, for $\xi=1$ one can write the form factor in Eq. (10) as

$$
\left|F_{\zeta \zeta^{\prime}}(u)\right|^{2} \equiv\left|F_{n s_{z}}^{\lambda 1}(u)\right|^{2}=\frac{1}{2} e^{-u}\left[\eta_{+}^{2} L_{n}(u)+\eta_{-}^{2} L_{n-1}(u)\right]^{2},
$$

for $n>0$, and $\left|F_{0 s_{z}}^{1}(u)\right|^{2}=e^{-u}$ for $n=0$. This expression is identical to that of Ref. [9] in which, however, the gap has a different origin. The factor $\left(y_{\zeta}-y_{\zeta^{\prime}}\right)^{2}$ is equal to $\ell_{c}^{4} q^{2} \sin ^{2} \varphi$. Now using polar coordinates, $\sum_{k_{x}} \rightarrow S_{0} / 2 \pi \ell_{c}^{2}$ and $\sum_{\mathbf{q}} \rightarrow\left(S_{0} / 4 \pi^{2}\right) \int q d q \int d \varphi=\left[S_{0} /\left(2 \pi \ell_{c}\right)^{2}\right] \int d u \int d \varphi$, Eq. (9) takes the form

$$
\sigma_{x x}^{\mathrm{col}}=\frac{e^{2}}{h} \frac{\beta n_{i} U_{0}^{2}}{4 u_{c} \hbar \omega_{c}} \sum_{n, s_{z}, \lambda, \xi} M_{n s_{z}}^{\lambda \xi} E_{n s_{z}}^{\lambda \xi} f\left(E_{n s_{z}}^{\lambda \xi}\right)\left[1-f\left(E_{n s_{z}}^{\lambda \xi}\right)\right],
$$

where $u_{c}=\ell_{c}^{2} k_{s}^{2} / 2$ and

$$
M_{n s_{z}}^{\lambda \xi}=\int_{0}^{\infty} u\left|F_{n s_{z}}^{\lambda \xi}(u)\right|^{2} d u .
$$

Observing that in the approximate derivation of Eq. (20) the quantity $U_{0} / \kappa_{s}$ is equivalent to the strength $V_{0}$ of Ref. [14], we see that $n_{i} U_{0}^{2} / 4 u_{c} \hbar \omega_{c} \propto 1 / A$ and consequently $\sigma_{x x}^{\text {col }}$ is inversely proportional to the constant $A$ introduced above.

In Eq. (20) the summation over $\xi$ takes into account the two valleys. Similar to the case of the Hall conductivity, this summation is necessary because the energy spectra in the two valleys differ in the zeroth level. The integration in Eq. (21) is carried out analytically using the orthogonality of the polynomials $L_{n}(u)$ and their recurrence relation $(n+1) L_{n+1}(u)-(2 n+1-u) L_{n}(u)+n L_{n-1}(u)=0 . \quad$ The result is

$$
M_{n, s_{z}}^{\lambda, 1}=\frac{1}{4}\left[(2 n+1) \eta_{+}^{4}-2 n \eta_{+}^{2} \eta_{-}^{2}+(2 n-1) \eta_{-}^{4}\right] .
$$

A similar expression can be derived for $\xi=-1$. When $E_{z}=$ $\lambda_{\text {so }}=0$, the expression for $M_{n s_{\mathrm{z}}}^{\lambda 1}$ reduces to $2 n / 4$, which means that the minima of the diagonal conductivity $\sigma_{x x}$ occur at odd filling factors $v=2 n+1$ in accord with Ref. [21]. Recall that in common semiconductors the minima occur at even filling factors $v=2 n$, and that $\sigma_{x x} \propto(2 n+1)$.

In Fig. 6 we plot the longitudinal conductivity given by Eqs. (20) as well as the Fermi level and density of states (DOS) as functions of the magnetic field $B$. The blue dashed (red solid) curves indicate the results in the absence (presence) of the electric field. One sees that the LL splitting by a finite electric field has doubled the number of the Fermi level jumps and the peaks in the DOS and Shubnikov-de Haas oscillations of the longitudinal conductivity. As expected, the conductivity resonances coincide with the peaks of the DOS and its value drops, for finite field $E_{z}$, when the Fermi level passes through two successive LLs or sublevels. This can be clearly seen by tracing out the vertical dashed lines. The longitudinal conductivity is also shown in Fig. 6(b) for a larger broadening 

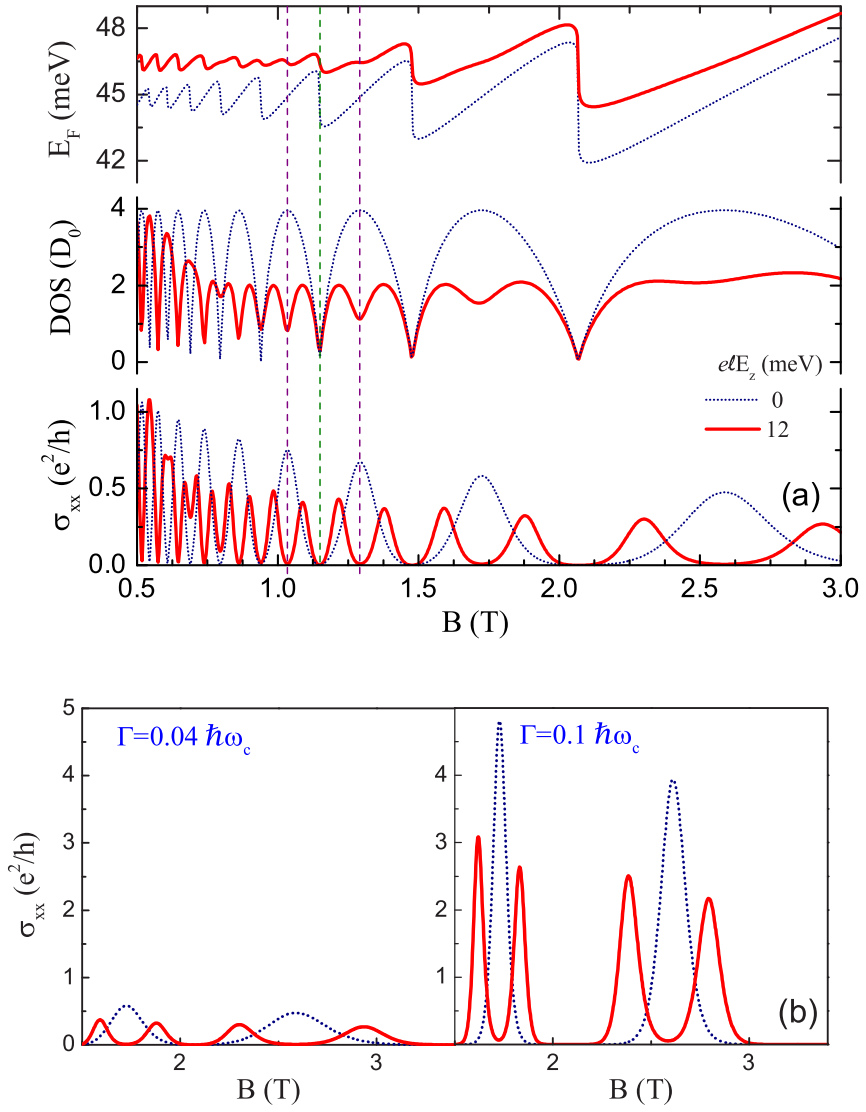

FIG. 6. (Color online) (a) Fermi level, density of states near the Fermi level, and longitudinal conductivity vs magnetic field $B$ for $E_{z}=0$ (dotted curves) and $E_{z}=12 \mathrm{mV} / \ell$ (solid curves). The broadening is $\Gamma=0.04 \hbar \omega_{c}, T=2 \mathrm{~K}$, and the electron density $N_{e}=5 \times 10^{11} / \mathrm{cm}^{2}$. The doubling of the $\sigma_{x x}$ peaks for $E_{z} \neq 0$ directly correlates with that of the DOS and the intra- $L L$ transitions of the Fermi level (see vertical lines). (b) Longitudinal conductivity for $\Gamma=0.04 \hbar \omega_{c}$ and $\Gamma=0.1 \hbar \omega_{c}$ in the range $1.5 \leqslant B \leqslant 3.5 \mathrm{~T}$.

$\Gamma=0.1 \hbar \omega_{c}$. The oscillation peaks have become sharp but more narrow compared to those in Fig. 6(a).

Another measurable quantity is the magnetoresistivity tensor given, in terms of the conductivity tensor, by

$$
\rho=\left[\begin{array}{ll}
\rho_{x x} & \rho_{x y} \\
\rho_{y x} & \rho_{y y}
\end{array}\right]=\frac{1}{S}\left[\begin{array}{cc}
\sigma_{y y} & -\sigma_{x y} \\
-\sigma_{y x} & \sigma_{x x}
\end{array}\right],
$$

where $S=\sigma_{x x} \sigma_{y y}-\sigma_{x y} \sigma_{y x}$. Since the system is assumed to be isotropic, the longitudinal components $\rho_{x x}$ and $\rho_{y y}$ are equal. The results obtained for the longitudinal resistivity $\rho_{x x}$, as a function of the field $B$ and the electron density $n_{e}$ are summarized in Fig. 7 for $E_{z}=0$ in (a) and $e \ell E_{z}=12 \mathrm{meV}$ in (b). The white triangular strip near the center of each figure, at the vicinity of $N_{e}=0$, shows a region in which the resistivity diverges because simply no carriers exist in the conduction or valence band to contribute to the conductivity. In the other regions the resistivity peaks appear as a fan diagram [22,23] whose blades broaden with increasing $B$ corresponding to the broadening of the DOS; see Fig. 6(a). On the other hand, by decreasing $B$ one sees numerous superficial peaks that disappear gradually when $B$ vanishes. The reason is that for weak fields $B$ the number of LLs near the Fermi
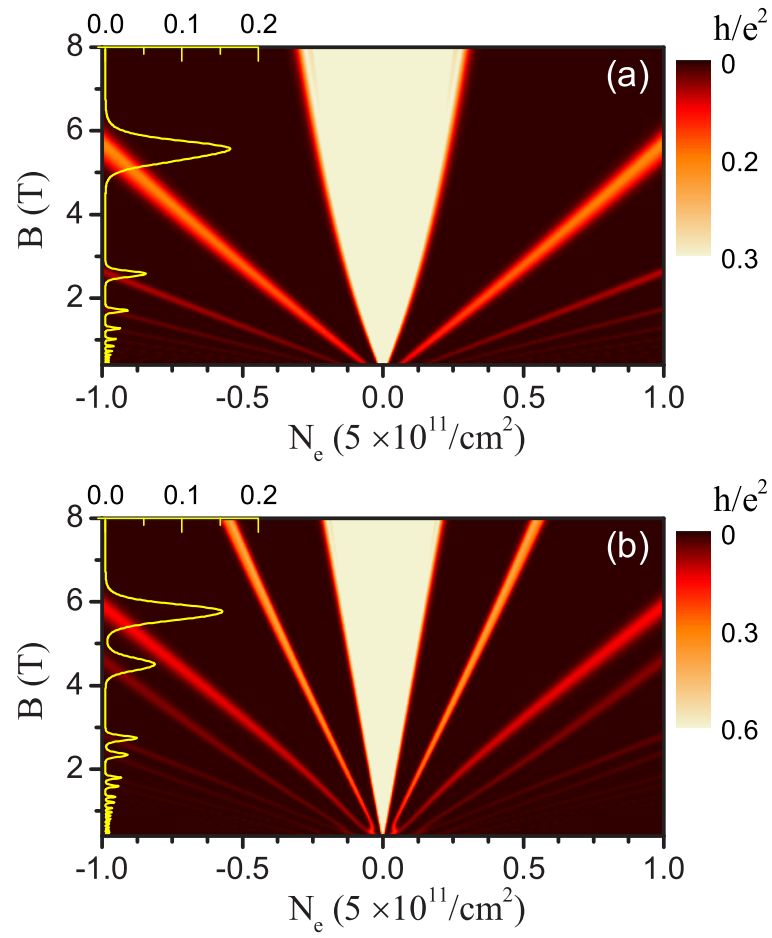

FIG. 7. (Color online) $\left(B, n_{e}\right)$ fan diagram of the longitudinal resistivity $\rho_{x x}$, in units of $\hbar / e^{2}$, and the curve of $\rho_{x x}$ (upper $x$ axis) vs field $B$ (left $y$ axis). Panel (a) is for $E_{z}=0$ and panel (b) for $e \ell E_{z}=12 \mathrm{meV}$. Further, $\Gamma=0.1 \hbar \omega_{c}$ and $N_{e}=5 \times 10^{11} / \mathrm{cm}^{2}$. In the bright region $\rho_{x x}$ diverges; the color scale appears on the right.

level participating in the longitudinal conductivity increases. Another notable feature of the fan diagram is that the number of peaks in $\rho_{x x}$ is doubled for finite field $E_{z}$ due to the lifting of the spin degeneracy.

In Fig. 8 we plot the longitudinal and transverse components of the conductivity and resistivity tensors, shown respectively in (a) and (b), as function of the electron density $N_{e}$ for e $\ell E_{z}=0 \mathrm{meV}, B=3 \mathrm{~T}$, and the dimensionless parameter $A=150$ (the sample is assumed to be very clean). As usual, the transition regions between the plateaus of $\rho_{x y}$ are accompanied by peaks in $\rho_{x x}$; the distance between the peaks, shown in (b), is $n_{0}=g_{s} g_{v} B / \phi_{0}$ which is in agreement with the observations on graphene samples of Ref. [20]. The heights of the first three plateaus of $\rho_{x y}$ are $1 / 2,1 / 6,1 / 10$. A deep minimum is seen in $\rho_{x y}=\sigma_{y x} /\left(\sigma_{y x}^{2}+\sigma_{x x}^{2}\right)$ at low electron densities and occurs, as shown, when the longitudinal conductivity component $\sigma_{x x}$ exceeds the component $\sigma_{y x}$. Similar deeps have been experimentally observed in graphene samples [24,25].

Finally, in Fig. 9 we show the same plots as in Fig. 8 but for $e \ell E_{z}=12 \mathrm{meV}$. As shown in Fig. 8(b), the distance between the peaks of $\rho_{x x}$ is $n_{0}=g_{s} g_{v} B / \phi_{0}$. The reason is that in strong magnetic fields the LL spacing is very large and the peaks of the DOS are well separated so that the overlap between them vanishes. In this case each LL has $g_{s} g_{v} / 2 \pi \ell_{c}^{2}=g_{s} g_{v} B / \phi_{0}$ states, per area $S_{0}$, that can be filled by electrons before the next LL starts to fill. However, the regularity of the peaks is weakened or destroyed when an external electric field is present because this field lifts the spin degeneracy and makes adjacent peaks of the DOS move 

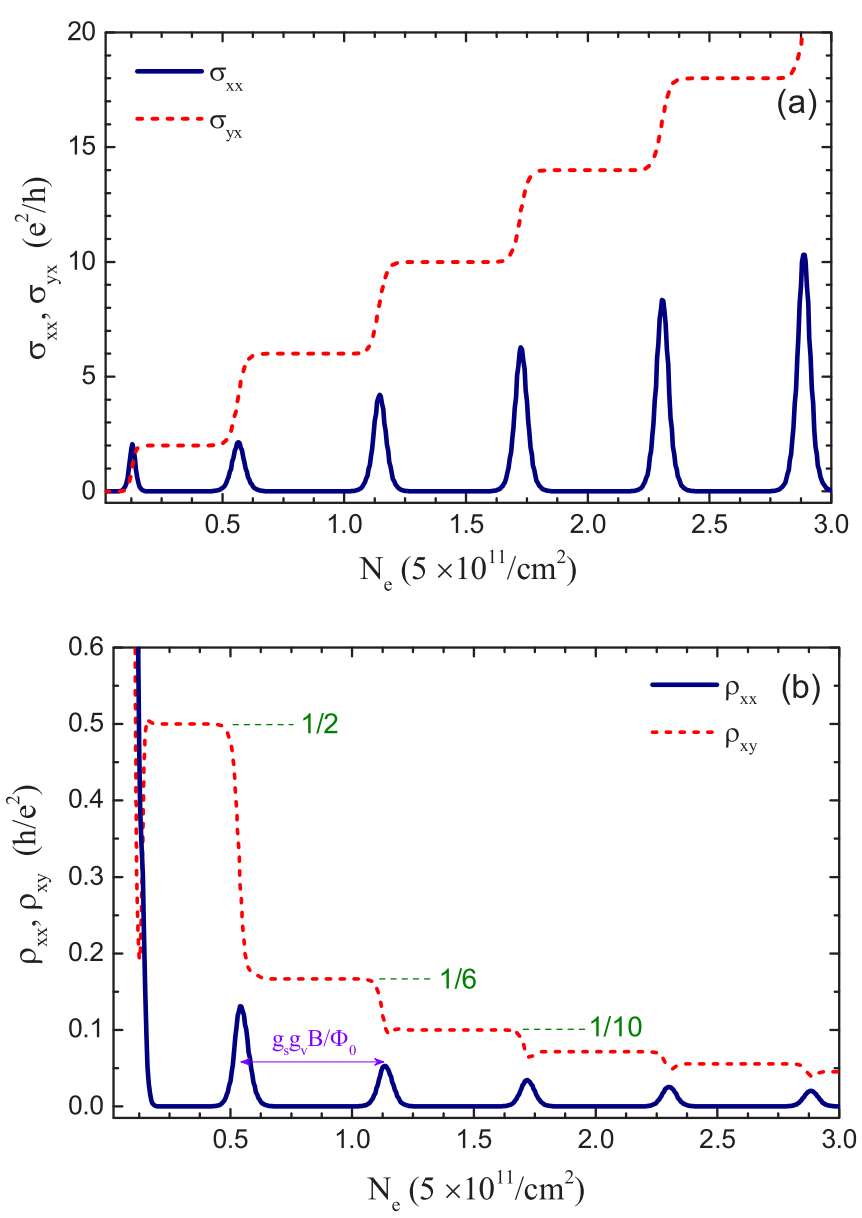

FIG. 8. (Color online) (a) The Hall conductivity $\sigma_{y x}$ and the longitudinal component $\sigma_{x x}$ as a function of the electron density for $T=2 \mathrm{~K}, \Gamma=0.1 \hbar \omega_{c}, B=3 \mathrm{~T}$, and $E_{z}=0$. (b) The Hall $\left(\rho_{y x}\right)$ and longitudinal $\left(\rho_{x x}\right)$ resistivities for the same parameters as in (a). The dent in $\rho_{x x}$ at low electron density is due to the fact that $\sigma_{y x}<\sigma_{x x}$. The same dents are seen in the experimental results of Refs. [24] and [25].

closer to each other or overlap. Figure 9 shows this effect for $e \ell E_{z}=12 \mathrm{meV}$. As a result, the peaks of $\rho_{x x}$ are doubled and their regularity is broken. As expected, the electric field also doubles the number of the plateaus in $\rho_{x y}$ shown here at heights $1 / 2,1 / 4,1 / 6,1 / 8,1 / 10$.

\section{SUMMARY}

We studied the transport coefficients of freestanding monolayer silicene subject to a quantizing magnetic field. Due to silicene's buckled structure, the gap between the conduction and valence bands, due to the strong SOI, can be further controlled by an external electric field; see Fig. 1(b). It was shown that the combined action of the electric field and strong SOI reduces the degeneracy of the LLs by a factor of 2, facilitates intra- $L L$ transitions, and results in integer and half-integer quantum Hall plateaus. As detailed in the text, the latter plateaus are more sensitive to disorder and thermal broadening than the former; cf. Fig. 5. Moreover, the electric field doubles the peaks of the Shubnikov-de Haas oscillations of the longitudinal conductivity. The deep minima
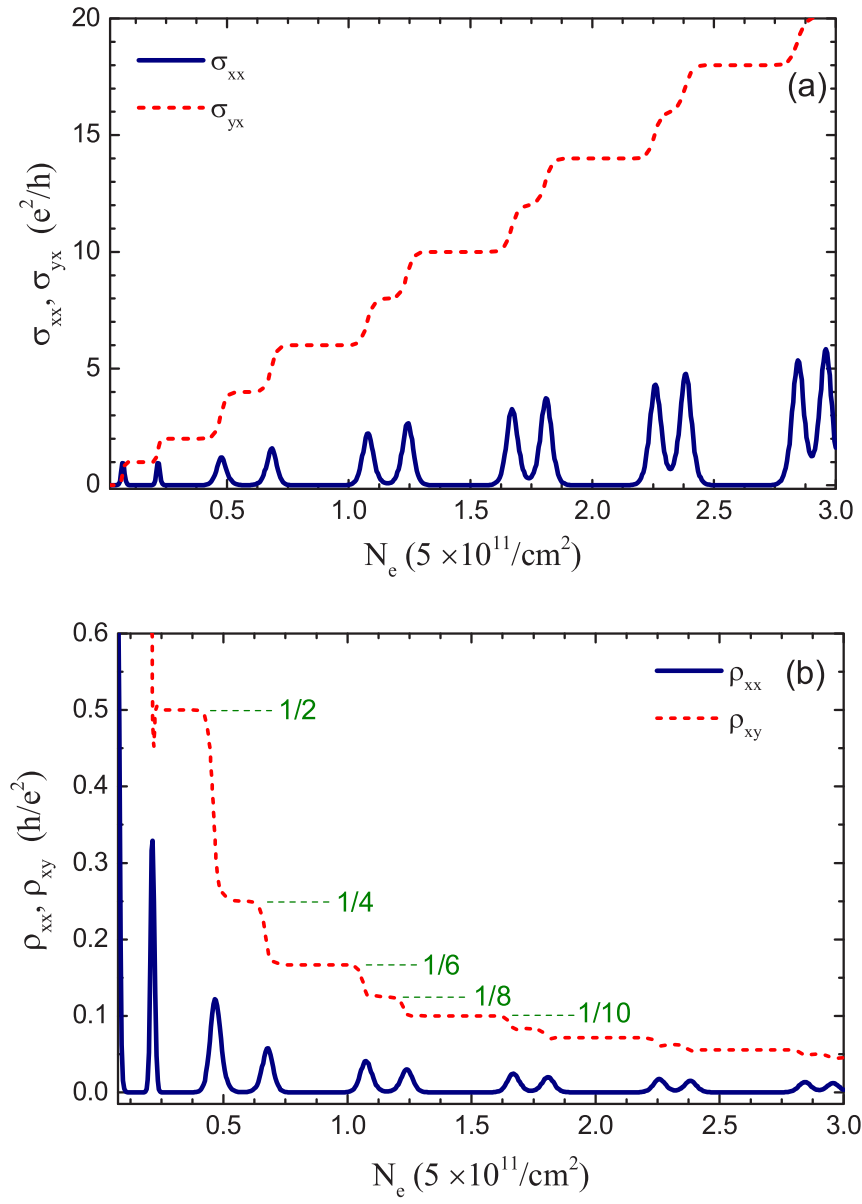

FIG. 9. (Color online) The same as in Fig. 8 but for $e \ell E_{z}=$ $12 \mathrm{meV}$.

in these oscillations are accompanied by Fermi level jumps and the peaks coincide with the usual singularities of the DOS. Mapping the magnetoresistivity in terms of the electron density and magnetic field results in a fan diagram whose blades are broadened with increasing magnetic field. A doubling of the peaks is also predicted for the resistivity when a perpendicular homogeneous electric field is present.

\section{ACKNOWLEDGMENTS}

Our work was supported by the Flemish Science Foundation (FWO-VI), the Methusalem Foundation of the Flemish Government, and the Canadian NSERC Grant No. OGP0121756.

\section{APPENDIX: DERIVATION OF THE HALL CONDUCTIVITY}

Below we outline the derivation of the Hall conductivity. We first consider the case $\xi=1$. Using Eq. (11), the contribution of the $K$ valley can be expressed as

$$
\sigma_{y x}^{K}(0)=\frac{i \hbar e^{2}}{S_{0}} \sum_{k_{x}} \sum_{n n^{\prime} \lambda \lambda^{\prime}} \sum_{s_{z} s_{z}^{\prime}} \frac{\left(f_{n s_{z}}^{\lambda 1}-f_{n^{\prime} s_{z}^{\prime}}^{\lambda^{\prime} 1}\right) P_{n n^{\prime}, s_{z} s_{z}^{\prime}}^{\lambda \lambda^{\prime}} .}{\left(E_{n s_{z}}^{\lambda 1}-E_{n^{\prime} s_{z}^{\prime}}^{\lambda^{\prime} 1}\right)^{2}} .
$$

For simplicity, in what follows we drop the superscript 1 from the Fermi function and energy. In addition, in order to sum over 
the band index $\lambda$ and eliminating it we express the energy as $E_{n s_{z}}^{\lambda}=\lambda \epsilon_{n s_{z}}$; cf. Eq. (3). With the help of the matrix elements given in Eq. (13) and by considering the spin orthogonality $\delta_{s_{z}, s_{z}^{\prime}}$, Eq. (A1) yields

$$
\sigma_{y x}^{K}=\sigma_{0 K}-C \sum_{k_{x}} \sum_{n=1, s_{z}} \sum_{\lambda \lambda^{\prime}}\left(I_{n s_{z}}^{\lambda \lambda^{\prime}}+I_{n s_{z}}^{\lambda^{\prime} \lambda}\right),
$$

where $\sigma_{0 K}$ is the contribution from the zeroth LL, $C=$ $e^{2} v_{F}^{2} / \hbar \omega^{2} S_{0}$, and

$$
\begin{aligned}
I_{n s_{z}}^{\lambda \lambda^{\prime}}= & \frac{f_{n+1 s_{z}}^{\lambda}-f_{n s_{z}}^{\lambda^{\prime}}}{\left(\lambda \epsilon_{n+1 s_{z}}-\lambda^{\prime} \epsilon_{n s_{z}}\right)^{2}} \\
& \times \frac{\left(e_{s_{z},+}+\lambda^{\prime} \epsilon_{n s_{z}}\right)\left(-e_{s_{z},+}+\lambda \epsilon_{n+1 s_{z}}\right)}{4 \lambda \lambda^{\prime} \epsilon_{n+1 s_{z}} \epsilon_{n s_{z}}} .
\end{aligned}
$$

Summing over $\lambda$ and $\lambda^{\prime}$, Eq. (A2) can be written as

$$
\sigma_{y x}^{K}(0)=\sigma_{0 K}-2 C \sum_{k_{x}} \sum_{n=1, s_{z}}\left(I_{n s_{z}}^{++}+I_{n s_{z}}^{--}+I_{n s_{z}}^{+-}+I_{n s_{z}}^{-+}\right) \text {. }
$$

We calculate the sum $I_{n s_{z}}^{++}+I_{n s_{z}}^{+-}$using Eq. (A3). The Fermi function $f_{n+1 s_{z}}^{+}$enters with a prefactor given by

$$
\begin{aligned}
& \frac{\epsilon_{n+1 s_{z}}-e_{s_{z},+}}{4 \epsilon_{n+1 s_{z}} \epsilon_{n s_{z}}}\left[\frac{e_{s_{z},+}+\epsilon_{n s_{z}}}{\left(\epsilon_{n+1 s_{z}}-\epsilon_{n s_{z}}\right)^{2}}-\frac{e_{s_{z},+}-\epsilon_{n s_{z}}}{\left(\epsilon_{n+1 s_{z}}+\epsilon_{n s_{z}}\right)^{2}}\right] \\
& \quad=n+\frac{1}{2}+\frac{e_{s_{z},+}}{2 \epsilon_{n+1 s_{z}}} .
\end{aligned}
$$

The functions $f_{n s_{z}}^{+}, f_{n s_{z}}^{-}$, and $f_{n+1 s_{z}}^{-}$enter in a similar way. We obtain

$$
\begin{aligned}
\sum_{\lambda \lambda^{\prime}= \pm} I_{n s_{z}}^{\lambda \lambda^{\prime}}= & \left(n+\frac{1}{2}\right)\left(f_{n+1 s_{z}}^{+}+f_{n+1 s_{z}}^{-}-f_{n s_{z}}^{+}-f_{n s_{z}}^{-}\right) \\
& +\frac{e_{s_{z},+}}{2}\left[\frac{f_{n+1 s_{z}}^{+}-f_{n+1 s_{z}}^{-}}{\epsilon_{n+1 s_{z}}}-\frac{f_{n s_{z}}^{+}-f_{n s_{z}}^{-}}{\epsilon_{n s_{z}}}\right] .
\end{aligned}
$$

Now, by substituting $\sum_{k_{x}} \rightarrow L_{x} \int_{0}^{L_{y} / \ell_{c}^{2}} d k_{x} / 2 \pi$ and using Eq. (A6) the contribution of the $n \geqslant 1$ LLs, i.e., the second term in Eq. (A2), is given by $\sigma_{K}+\delta \sigma_{K}$ where

$$
\begin{gathered}
\sigma_{K}=\frac{e^{2}}{h} \sum_{n=1, s_{z}}\left(n+\frac{1}{2}\right)\left(f_{n s_{z}}^{+}+f_{n s_{z}}^{-}-f_{n+1 s_{z}}^{+}-f_{n+1 s_{z}}^{-}\right), \\
\delta \sigma_{K}=\frac{e^{2}}{2 h} \sum_{n=1, s_{z}} e_{s_{z},+}\left[\frac{f_{n s_{z}}^{+}-f_{n s_{z}}^{-}}{\epsilon_{n s_{z}}}-\frac{f_{n+1 s_{z}}^{+}-f_{n+1 s_{z}}^{-}}{\epsilon_{n+1 s_{z}}}\right] .
\end{gathered}
$$

Similarly, the contribution from the zeroth LL is

$\sigma_{0 K}=\frac{e^{2}}{h} \sum_{s_{z}} f_{0 s_{z}}-\frac{1}{2}\left(f_{1 s_{z}}^{+}+f_{1 s_{z}}^{-}\right)+\frac{e_{s z+}}{2 \epsilon_{1 s_{z}}}\left(f_{1 s_{z}}^{+}-f_{1 s_{z}}^{-}\right)$.

For the $K^{\prime}$ valley one follows the same steps. The resulting expressions analogous to Eqs. (A7)-(A9) are

$$
\sigma_{K^{\prime}}=\frac{e^{2}}{h} \sum_{n=1, s_{z}}\left(n+\frac{1}{2}\right)\left(f_{n s_{z}}^{+}+f_{n s_{z}}^{-}-f_{n+1 s_{z}}^{+}-f_{n+1 s_{z}}^{-}\right),
$$

$$
\begin{aligned}
\delta \sigma_{K^{\prime}} & =-\frac{e^{2}}{2 h} \sum_{n=1, s_{z}} e_{s_{z}},-\left[\frac{f_{n s_{z}}^{+}-f_{n s_{z}}^{-}}{\epsilon_{n s_{z}}}-\frac{f_{n+1 s_{z}}^{+}-f_{n+1 s_{z}}^{-}}{\epsilon_{n+1 s_{z}}}\right] \\
\sigma_{0 K^{\prime}} & =\frac{e^{2}}{h} \sum_{s_{z}} f_{0 s_{z}}-\frac{1}{2}\left(f_{1 s_{z}}^{+}+f_{1 s_{z}}^{-}\right)-\frac{e_{s z-}}{2 \epsilon_{1 s_{z}}}\left(f_{1 s_{z}}^{+}-f_{1 s_{z}}^{-}\right)
\end{aligned}
$$

Writing explicitly the sum over $s_{z}$ one sees that the correction terms $\delta \sigma_{K}$ and $\delta \sigma_{K^{\prime}}$ cancel each other if there is identical occupancy of the degenerate states.
[1] G. G. Guzmán-Verri and L. C. Lew Yan Voon, Phys. Rev. B 76, 075131 (2007); S. Lebègue and O. Eriksson, ibid. 79, 115409 (2009).

[2] P. Vogt, P. De Padova, C. Quaresima, J. Avila, E. Frantzeskakis, M. C. Asensio, A. Resta, B. Ealet, and G. Le Lay, Phys. Rev. Lett. 108, 155501 (2012); A. Fleurence, R. Friedlein, T. Ozaki, H. Kawai, Y. Wang, and Y. Yamada-Takamura, ibid. 108, 245501 (2012).

[3] D. Chiappe, E. Scalise, E. Cinquanta, C. Grazianetti, B. v. Broek, M. Fanciulli, M. Houssa, and A. Molle, Adv. Mater. 26, 2096 (2014).

[4] Z. Ni, Q. Liu, K. Tang, J. Zheng, J. Zhou, Rui Qin, Z. Gao, D. Yu, and Jing Lu, Nano Lett. 12, 113 (2012); L. Chen, H. Li, B. Feng, Z. Ding, J. Qiu, P. Cheng, K. Wu, and S. Meng, Phys. Rev. Lett. 110, 085504 (2013); M. Neek-Amal, A. Sadeghi, G. R. Berdiyorov, and F. M. Peeters, Appl. Phys. Lett. 103, 261904 (2013).
[5] C.-C. Liu, W. Feng, and Y. Yao, Phys. Rev. Lett. 107, 076802 (2011); X.-L. Zhang, L.-F. Liu, and W.-M. Liu, Sci. Rep. 3, 2908 (2013); M. Tahir and U. Schwingenschlgl, Appl. Phys. Lett. 102, 162412 (2013).

[6] M. Ezawa, Phys. Rev. Lett. 109, 055502 (2012).

[7] A. Kara, H. Enriquez, A. P. Seitsonen, L. C. Lew Yan Voon, S. Vizzini, B. Aufray, and H. Oughaddoub, Surf. Sci. 67, 1 (2012).

[8] L. Jiang, Y. Zheng, H. Li, and H. Shen, Nanotechnology 21, 145703 (2010).

[9] P. M. Krstajić and P. Vasilopoulos, Phys. Rev. B 86, 115432 (2012).

[10] Kh. Shakouri, P. Vasilopoulos, V. Vargiamidis, and F. M. Peeters, Phys. Rev. B 90, 125444 (2014).

[11] C.-C. Liu, H. Jiang, and Y. Yao, Phys. Rev. B 84, 195430 (2011).

[12] B. Van Dupen, P. Vasilopoulos, and F. M. Peeters, Phys. Rev. B 90, 035142 (2014).

[13] C. J. Tabert and E. J. Nicol, Phys. Rev. Lett. 110, 197402 (2013). 
[14] Y. Zheng and T. Ando, Phys. Rev. B 65, 245420 (2002).

[15] M. Charbonneau, K. M. Van Vliet, and P. Vasilopoulos, J. Math. Phys. 23, 318 (1982).

[16] P. Vasilopoulos, Phys. Rev. B 32, 771 (1985).

[17] P. M. Krstajić and P. Vasilopoulos, Phys. Rev. B 83, 075427 (2011).

[18] A. F. Young, C. R. Dean, L. Wang, H. Ren, P. Cadden-Zimansky, K. Watanabe, T. Taniguchi, J. Hone, K. L. Shepard, and P. Kim, Nat. Phys. 8, 550 (2012).

[19] Igor F. Herbut, Phys. Rev. B 75, 165411 (2007).

[20] K. S. Novoselov, A. K. Geim, S. V. Morozov, D. Jiang, M. I. Katsnelson, I. V. Grigorieva, S. V. Dubonos, and A. A. Firsov, Nature (London) 438, 197 (2005).

[21] V. P. Gusynin and S. G. Sharapov, Phys. Rev. Lett. 95, 146801 (2005).
[22] L. A. Ponomarenko, R. V. Gorbachev, G. L. Yu, D. C. Elias, R. Jalil, A. A. Patel, A. Mishchenko, A. S. Mayorov, C. R. Woods, J. R. Wallbank, M. Mucha-Kruczynski, B. A. Piot, M. Potemski, I. V. Grigorieva, K. S. Novoselov, F. Guinea, V. I. Falko, and A. K. Geim, Nature (London) 497, 594 (2013).

[23] C. R. Dean, L. Wang, P. Maher, C. Forsythe, F. Ghahari, Y. Gao, J. Katoch, M. Ishigami, P. Moon, M. Koshino, T. Taniguchi, K. Watanabe, K. L. Shepard, J. Hone, and P. Kim, Nature (London) 497, 598 (2013).

[24] Yuanbo Zhang, Yan-Wen Tan, Horst L. Stormer, and Philip Kim, Nature (London) 438, 201 (2005).

[25] C. R. Dean, A. F. Young, P. Cadden-Zimansky, L. Wang, H. Ren, K. Watanabe, T. Taniguchi, P. Kim, J. Hone, and K. L. Shepard, Nat. Phys. 7, 693 (2011). 